CPTH-S055.0500

\title{
Comments on Perturbative Dynamics of Non-Commutative Yang-Mills Theory
}

\author{
Adi Armoni \\ Centre de Physique Théorique de l'École Polytechnique \\ 91128 Palaiseau Cedex, France \\ armoni@cpht.polytechnique.fr
}

\begin{abstract}
We study the $U(N)$ non-commutative Yang-Mills theory at the one-loop approximation. We check renormalizability and gauge invariance of the model and calculate the one-loop beta function. The interaction of the $S U(N)$ gauge bosons with the $U(1)$ gauge boson plays an important role in the consistency check. In particular, the $S U(N)$ theory by itself is not consistent. We also find that the $\theta \rightarrow 0$ limit of the $U(N)$ theory does not converge to the ordinary $S U(N) \times U(1)$ commutative theory, even at the planar limit. Finally, we comment on the UV/IR mixing.
\end{abstract}




\section{Introduction and Conclusions}

Non-commutative gauge field theories lately attracted a lot of attention, mainly due to the discoveries of their relation to string theory [1]. It was also found that the perturbative structure of these theories has an interesting pattern. It was shown [2], in the case of scalar theory, that planar diagrams of the non-commutative theory are the same as planar diagrams of ordinary commutative theory, up to global phases. For an earlier related work see ref.[3]. It was then suggested [0] that non-planar graphs are UV finite, due to the oscillatory Moyal phase which regulates the integrals. It was found later [5] that these contributions actually lead to divergences, which were interpreted as infra-red divergences. These contributions are singular in the $\theta \rightarrow 0$ limit and they occur also in gauge theories [6, 7].

This paper is devoted to the study of $U(N)$ non-commutative gauge theory. The $U(1)$ case was already studied by several authors [8, 9, 10, 6, [] The renormalization of the model, at the one loop approximation, was studied first in [8]. The UV/IR mixing, in the $U(1)$ case was studied by [6] and [7]. Related works about perturbative dynamics of non-commutative field theories are [11, 12, 13, 14, 15, 16, 17, 18, 19, 20, 21, 22, 23. Perturbative aspects of non-commutative field theories from string theory were discussed in $24,25,26,27,28,29]$.

The non-commutative $U(N)$ Yang-Mills action is

$$
\int d^{4} x \operatorname{tr}-\frac{1}{2 g^{2}} F_{\mu \nu} \star F^{\mu \nu}
$$

where $F_{\mu \nu}$ is

$$
F_{\mu \nu}=\partial_{\mu} A_{\nu}-\partial_{\nu} A_{\mu}-i\left(A_{\mu} \star A_{\nu}-A_{\nu} \star A_{\mu}\right)
$$

and $A_{\mu}$ is a $N \times N$ matrix. The $\star$-product between two functions $f$ and $g$ is defined by

$$
f \star g(x)=\left.e^{\frac{i}{2} \theta^{\mu \nu} \partial_{\mu}^{(\xi)} \partial_{\nu}^{(\eta)}} f(x+\xi) g(x+\eta)\right|_{\xi, \eta \rightarrow 0} .
$$

The action (1) is invariant under $U(N)$ gauge transformation

$$
\delta_{\lambda} A_{\mu}=\partial_{\mu} \lambda-i\left(A_{\mu} \star \lambda-\lambda \star A_{\mu}\right) .
$$

The gauge transformation (픈 is different from the commutative gauge transformation in the sense that it mixes the $U(1)$ gauge boson with the $S U(N)$ 
gauge bosons. In fact, the non-commutative Yang-Mills action (四) also mixes the $U(1)$ and the $S U(N)^{\prime} s$. It cannot be written as a sum of a $S U(N)$ and a $U(1)$ theories as the ordinary YM theory, since there are interaction terms between the $S U(N)$ gluons and the $U(1)$ 'photon'. In order to demonstrate this point we list in figure 1 below the Feynman rules which describe the 3 -gluons interaction (the full list of Feynman rules is written in Appendix A)

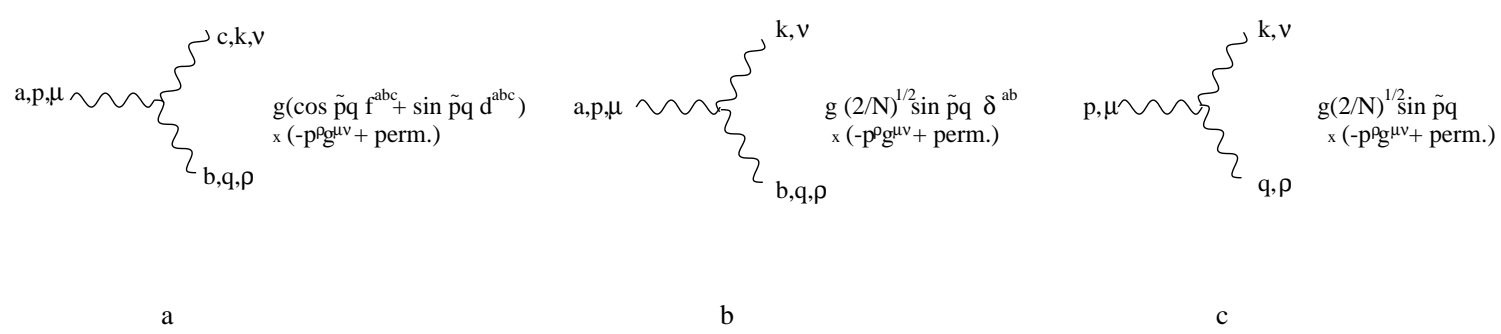

Figure 1: Contributions to the 3 gluons vertex. a). $S U(N)-S U(N)-S U(N)$ interaction. b). $S U(N)-S U(N)-U(1)$ interaction. c). $U(1)-U(1)-U(1)$ interaction.

The complicated structure of the action (11) raises the question of gauge invariance and consistency of the non-commutative Yang-Mills theory at the quantum level. The action (11) consists of many interaction terms with a single coupling $g$ - due to gauge invariance. It is not clear, a-priori, that the relations among the various couplings in the action is kept at the quantum level. There are two limits of the theory which hint that the full $U(N)$ gauge invariance might be broken. The first limit is $\theta \rightarrow 0$. In this case, the theory is expected to reduce to the ordinary commutative theory. However, the commutative theory has a $S U(N) \times U(1)$ gauge symmetry and the $S U(N)$ coupling is not related to the $U(1)$ coupling by gauge symmetry. Moreover, at the quantum level the $S U(N)$ coupling runs and the $U(1)$ coupling is kept fixed. The second limit, is the planar limit. Since it looks as if the noncommutative theory and the commutative are identical at the planar level, 
the same question about the $U(1)$ coupling should be raised.

As we shall see the $U(N)$ gauge symmetry is not broken quantum mechanically. The renormalization procedure does not violate the relations between the various couplings (at least at the one loop level). The resolution of the puzzles mentioned above, is the following: the limit $\theta \rightarrow 0$ does not lead to the ordinary commutative theory. Though the resulting action looks like the ordinary YM action (note that the $U(1)$ and the $S U(N)$ seems to decouple in the $\theta \rightarrow 0$ limit, see figure 1b), the $U(1)$ and $S U(N)$ couplings have exactly the same beta function.

The fact that the limit $\theta \rightarrow 0$ of the $U(1)$ theory is singular was already pointed out in [7]. It was with relation to the non-planar contributions, which are manifestly singular in $\theta$. We claim, however, that the theory is not smooth in $\theta$ even in the planar limit. In this case, indeed the $S U(N)$ sector theory looks like the commutative theory, but the interaction of the $U(1)$ with the $U(N)^{\prime} s$ survives the limit. In particular, the $U(1)$ gauge coupling runs. Thus, the planar sector of the $\theta \rightarrow 0$ theory does not correspond to the planar sector of the commutative theory. In this way, the puzzle about $U(N)$ gauge invariance at the quantum level is also resolved.

The main results of the paper are the following: in section 2 we calculate the counter terms which are needed to regulate the divergences in the planar graphs of the $S U(N)$ and $U(1)$ gluons propagators. We find that they are the same and equal to the ordinary commutative counter term of the $S U(N)$ propagator. The non-planar contributions, however, are different. There is a non-planar finite contribution to the $U(1)$ propagator [7], but there is no such contribution for the $S U(N)$. In section 3 we calculate the counter terms of the various 3 gluons vertices. Our results in this section are similar to those of section 2. The divergent (planar) part of the various 3 gluons vertices is the same, but the finite (non-planar) part is different. Finally, in section 4, we calculate the beta function, discuss our results and more general cases where also matter fields are present.

We shall use the following conventions: capital letters $(A, B, C, \ldots)$ denote $U(N)$ indices, small letters $(a, b, c, \ldots)$ denote $S U(N)$ indices. The $U(1)$ generator is normalized as follows $t^{0}=\frac{1}{\sqrt{2 N}}$, such that $\operatorname{tr} t^{A} t^{B}=\frac{1}{2} \delta^{A B}$. Finally, $\left[t^{a}, t^{b}\right]=i f^{a b c} t^{c}$ and $\left\{t^{a}, t^{b}\right\}=\frac{1}{N} \delta^{a b}+d^{a b c} t^{c}$. Thus $d^{a b c}$ represents the symmetric tensor for the fundamental representation. In addition, we shall use the notation $\tilde{p}_{\mu}=\frac{1}{2} \theta_{\mu \nu} p^{\nu}$. 


\section{Corrections to the gluon propagator}

In order to check the renormalizability and gauge invariance at the quantum level, let us start with the one loop correction to the gluon propagator. The various contribution are drawn in figure 2 below

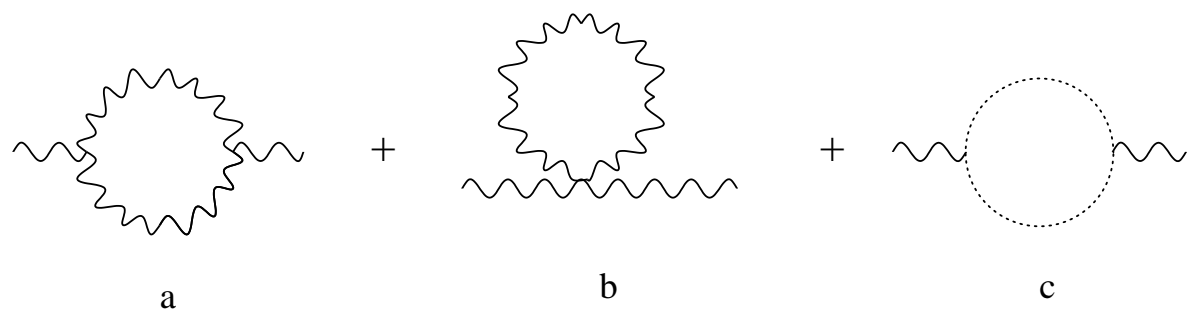

Figure 2: One loop corrections to the gluon propagator.

We consider first the case where the external legs carry $U(1)$ indices ('photons'). The calculation is a straightforward generalization of the calculations which were performed for the $U(1)$ non-commutative Yang-Mills theory [6, 7]. The three contributes are drawn in figure 2. Let us focus on diagram $2 \mathrm{a}$. The only difference in comparison with the $U(1)$ theory is that now all the $U(N)$ gluons can circulate in the loop. We will use the Feynman rules $1 \mathrm{~b}$ and 1c. We denote the external momentum by $p$ and the internal momentum by $q$. The resulting expression is

$$
\begin{aligned}
A^{\mu \nu}= & \frac{1}{2} \int \frac{d^{4} q}{(2 \pi)^{4}} \frac{-i}{q^{2}} \frac{-i}{(p+q)^{2}} \times T_{1} \times \\
& \left(g^{\mu \rho}(p-q)^{\sigma}+g^{\rho \sigma}(2 q+p)^{\mu}+g^{\sigma \mu}(-q-2 p)^{\rho}\right) \times \\
& \left(\delta_{\rho}^{\nu}(q-p)_{\sigma}+g_{\rho \sigma}(-2 q-p)^{\nu}+\delta_{\sigma}^{\nu}(q+2 p)_{\rho}\right), \\
T_{1}= & \frac{2 g^{2}}{N} \delta^{A B} \delta^{A B} \sin ^{2} \tilde{p} q
\end{aligned}
$$

By using the identity

$$
\sin ^{2} \tilde{p} q=\frac{1}{2}(1-\cos 2 \tilde{p} q)
$$


we can isolate the planar contribution which comes from the $\frac{1}{2}$, from the non-planar contribution (the cosine)

$$
T_{1}=g^{2} N+\text { non-planar term. }
$$

The planar part of the contribution is divergent and its value is exactly the same as the value of the divergent part of the gluon propagator in ordinary $S U(N)$ Yang-Mills theory. The same pattern occurs in the other diagrams in figure 2. Indeed, after the summation of the three diagrams in figure 2 we find that in order to cancel the divergent part of the $U(1)$ propagator the following counter term is needed

$$
\delta_{3}^{(1-1)}=\frac{g^{2} N}{(4 \pi)^{2}} \times \frac{5}{3} \times \frac{2}{\epsilon}
$$

where dimensional regularization was used and $\epsilon=4-d$. Note that the counter term does not depend on $\theta$. As long as $\theta$ is non zero, a counter term (9) is needed. Otherwise the $U(1)$ theory is free. Therefore, though the Feynman rules of the theory are smooth in $\theta$, the limit $\theta \rightarrow 0$ is singular.

For completeness let us quote the result for the finite part of the correction to the $U(1)$ propagator [7]. It is calculated by replacing the cosine of (7) by an exponent and by looking at the high momentum regime in the integrals of (5) and the two other diagrams in figure 2. The result is [7]

$$
A_{\text {finite }}^{\mu \nu}=-2 g^{2} N \int \frac{d^{4} q}{(2 \pi)^{4}} \frac{2 q^{\mu} q^{\nu}-g^{\mu \nu} q^{2}}{q^{4}} e^{i 2 \tilde{p} q} \sim g^{2} N \frac{\tilde{p}^{\mu} \tilde{p}^{\nu}}{\tilde{p}^{4}} \text {. }
$$

Note that this term is singular in $\theta$. When inserted into higher loops it behaves as ordinary infra-red divergences [5]. Thus, an effect which was originally due to high momentum turns out to be an IR effect. This is the UV/IR mixing which was found in [5].

Let us turn now to the calculation of the correction to the $S U(N)$ part of the gluon. Again, let us start with diagram 2a. The coupling of the $S U(N)$ bosons which circulate in the loop contains the symmetric tensor $d^{a b c}$. The integral is the same as (5), but $T_{1}$ is replaced by $T_{2}$

$$
T_{2}=g^{2}\left(f^{x y a} \cos \tilde{p} q+d^{x y a} \sin \tilde{p} q\right)\left(f^{x y b} \cos \tilde{p} q+d^{x y b} \sin \tilde{p} q\right)
$$


By using the $S U(N)$ identities (see Appendix B)

$$
\begin{aligned}
& f^{a x y} f^{b x y}=N \delta^{a b} \\
& d^{a x y} d^{b x y}=\left(N-\frac{4}{N}\right) \delta^{a b}
\end{aligned}
$$

$T_{2}$ can be written as

$$
T_{2}=g^{2} N\left(1-\frac{4}{N^{2}} \sin ^{2} \tilde{p} q\right) .
$$

Interestingly there is another contribution to the gluon propagator which doesn't occur in ordinary Yang-Mills theory, due to the existence of new vertices (fig. 1b). It is possible to exchange a $U(1)$ boson in half of the loop and $S U(N)$ boson in the other half. It contributes

$$
T_{2}^{\prime}=2 \times g^{2} \frac{2}{N} \sin ^{2} \tilde{p} q
$$

(the factor 2 in (15) represents two possible exchanges of the $U(1)$ ). Collecting the two terms (14) and (15), we find that the one loop correction to the $S U(N)$ propagator is exactly the same as in the commutative case. Thus the divergences can be compensated by the commutative counter term

$$
\delta_{3}^{(N-N)}=\frac{g^{2} N}{(4 \pi)^{2}} \times \frac{5}{3} \times \frac{2}{\epsilon} .
$$

Remarkably (91) is identical to (16) (except that (16) in needed also when $\theta=0$, in contrast to (9)). This fact is crucial to ensure gauge invariance of the model at the quantum level, as we shall see later.

It is interesting to note that the corrections to the $S U(N)$ propagator does not contain a non-planar finite part. Therefore, though the propagators are identical in their divergent part, they differ in their finite part. It is due to non-planar graphs which exist in the corrections to the $U(1)$ propagator but do not exist for the $S U(N)$ one.

\section{Corrections to the 3-gluons vertex}

In this section we calculate the one-loop corrections to the 3 gluons vertex. The relevant diagrams are listed in figure 3 below. The external momenta are $p_{1}, p_{2}, p_{3}$ with $p_{1}+p_{2}+p_{3}=0$. 


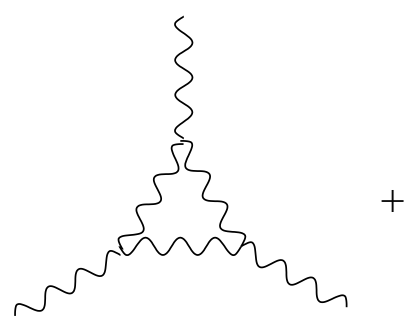

$\mathrm{a}$

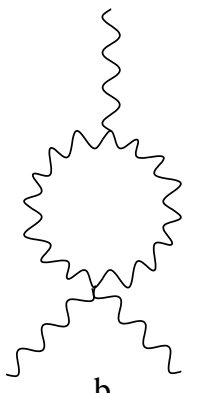

b

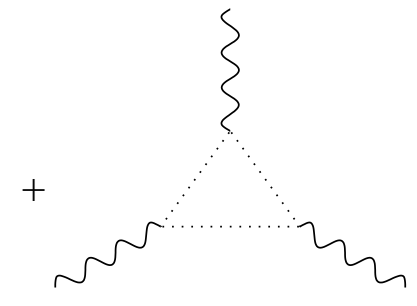

$\mathrm{c}$

Figure 3: One loop corrections to the 3-gluons vertex.

We begin with the simplest case in which all external legs are $U(1)^{\prime} s$. We focus on diagram 3a. The gluons which circulate in the loop belongs to $U(N)$. Similar calculations were made in refs. [6, 7].

$$
\begin{aligned}
M^{\mu \nu \rho}= & \frac{1}{2} \int \frac{d^{4} q}{(2 \pi)^{4}} \frac{-i}{q^{2}} \frac{-i}{\left(q-p_{2}\right)^{2}} \frac{-i}{\left(q+p_{1}\right)^{2}} \times V_{1} \times \\
& \left(g^{\sigma_{1} \mu}\left(q-p_{1}\right)^{\sigma_{2}}+g^{\mu \sigma_{2}}\left(2 p_{1}+q\right)^{\sigma_{1}}+g^{\sigma_{2} \sigma_{1}}\left(-p_{1}-2 q\right)^{\mu}\right) \times \\
& \left(\delta_{\sigma_{1}}^{\nu}\left(p_{2}+q\right)^{\sigma_{3}}+\delta_{\sigma_{1}}^{\sigma_{3}}\left(-2 q+p_{2}\right)^{\nu}+g^{\sigma_{3} \nu}\left(q-2 p_{2}\right)_{\sigma_{1}}\right) \times \\
& \left(g_{\sigma_{3} \sigma_{2}}\left(-2 q-p_{1}+p_{2}\right)^{\rho}+\delta_{\sigma_{2}}^{\rho}\left(p_{1}+q-p_{3}\right)_{\sigma_{3}}+\delta_{\sigma_{3}}^{\rho}\left(p_{3}+p_{2}-q\right)_{\sigma_{2}}\right), \\
V_{1}= & g^{3}\left(\frac{2}{N}\right)^{\frac{3}{2}} \delta^{X Y} \delta^{Y Z} \delta^{Z X} \sin \tilde{p}_{1} q \sin \tilde{p}_{2} q \sin \tilde{p}_{3}\left(q+p_{1}\right)
\end{aligned}
$$

By using the following identity

$$
\begin{aligned}
& \sin \tilde{p}_{1} q \sin \tilde{p}_{2} q \sin \tilde{p}_{3}\left(q+p_{1}\right)= \\
& -\frac{1}{4} \cos \tilde{p}_{3} p_{1}\left(\sin 2 \tilde{p}_{1} q+\sin 2 \tilde{p}_{2} q+\sin 2 \tilde{p}_{3} q\right) \\
& -\frac{1}{4} \sin \tilde{p}_{3} p_{1}\left(1-\cos 2 \tilde{p}_{1} q-\cos 2 \tilde{p}_{2} q+\cos 2 \tilde{p}_{3} q\right)
\end{aligned}
$$

we can isolate the divergent parts from the finite parts. The divergent part comes from the $-\frac{1}{4} \sin \tilde{p}_{3} p_{1}$ contribution. Since this part does not depend on $q$ it would lead to a contribution which is similar to the commutative case. 
The other diagrams in figure 3 are similar. Thus the needed counter term to cancel the divergent part of the $U(1)-U(1)-U(1)$ vertex is

$$
\delta_{1}^{(1-1-1)}=\frac{2 g^{2} N}{(4 \pi)^{2}} \times \frac{1}{4} \times \frac{4}{3} \times \frac{2}{\epsilon}=\frac{g^{2} N}{(4 \pi)^{2}} \times \frac{2}{3} \times \frac{2}{\epsilon}
$$

The finite part of the correction, which arise from the $-\frac{1}{4} \cos \tilde{p}_{3} p_{1} \times \sin 2 \tilde{p}_{i} q$ terms in (19), is calculated by replacing the sin by an exponent. The procedure is exactly the same as in the $U(1)$ case [7]. The result is

$$
\begin{aligned}
& M_{\text {finite }}^{\mu \nu \rho}= \\
& \quad g^{3} \sqrt{2 N} \cos \tilde{p}_{3} p_{1} \int \frac{d^{4} q}{(2 \pi)^{4}} \frac{1}{q^{6}}\left(4 q^{\mu} q^{\nu} q^{\rho}-q^{2}\left(q^{\mu} g^{\nu \rho}+q^{\nu} g^{\mu \rho}+q^{\rho} g^{\mu \nu}\right)\right) \times \\
& \quad\left(e^{i 2 \tilde{p}_{1} q}+e^{i 2 \tilde{p}_{2} q}+e^{i 2 \tilde{p}_{3} q}\right) \\
& \quad \sim g^{3} \sqrt{N} \cos \tilde{p}_{3} p_{1}\left(\frac{\tilde{p}_{1}^{\mu} \tilde{p}_{1}^{\nu} \tilde{p}_{1}^{\rho}}{\tilde{p}_{1}^{4}}+\frac{\tilde{p}_{2}^{\mu} \tilde{p}_{2}^{\nu} \tilde{p}_{2}^{\rho}}{\tilde{p}_{2}^{4}}+\frac{\tilde{p}_{3}^{\mu} \tilde{p}_{3}^{\nu} \tilde{p}_{3}^{\rho}}{\tilde{p}_{3}^{4}}\right)
\end{aligned}
$$

The calculation of the correction to the 3-gluons vertex when the external legs are in $S U(N)$, is a bit more complicated, as there are many contributions in the non-commutative case. The first contribution is when $S U(N)$ gluons circulate in the triangle of figure 3a. We will use the Feynman rules in figure 1a. The calculation of the diagram is performed by replacing $V_{1}$ by

$$
\begin{aligned}
V_{2}= & g^{3}\left(f^{a x y} \cos \tilde{p}_{1} q+d^{a x y} \sin \tilde{p}_{1} q\right) \times \\
& \left(f^{b y z} \cos \tilde{p}_{2} q+d^{b y z} \sin \tilde{p}_{2} q\right) \times \\
& \left(f^{c z x} \cos \tilde{p}_{3}\left(q+p_{1}\right)+d^{c z x} \sin \tilde{p}_{3}\left(q+p_{1}\right)\right)
\end{aligned}
$$

In order to simplify (22) we use the following $S U(N)$ identities (see Appendix B for derivation)

$$
\begin{aligned}
& f^{a x y} f^{b y z} f^{c z x}-f^{a x y} d^{b y z} d^{c z x} \\
& -d^{a x y} f^{b y z} d^{c z x}-d^{a x y} d^{b y z} f^{c z x}=2 N\left(1-\frac{3}{N^{2}}\right) f^{a b c}, \\
& d^{a x y} d^{b y z} d^{c z x}-d^{a x y} f^{b y z} f^{c z x} \\
& -f^{a x y} d^{b y z} f^{c z x}-f^{a x y} f^{b y z} d^{c z x}=2 N\left(1-\frac{3}{N^{2}}\right) d^{a b c},
\end{aligned}
$$


and trigonometric identities similar to (7). Hence, $V_{2}$ can be written as follows

$$
V_{2}=-g^{3}\left(f^{a b c} \cos \tilde{p}_{3} p_{1}+d^{a b c} \sin \tilde{p}_{3} p_{1}\right) \frac{N}{2}\left(1-\frac{3}{N^{2}}\right)+\text { other terms, }
$$

where 'other terms' means additional contributions which do not lead to divergences. Apart from the $V_{2}$ contribution, there is another contribution to the $S U(N)-S U(N)-S U(N)$ vertex. It is due to $S U(N)$ bosons flowing in two of the sides of the triangle in figure $3 \mathrm{a}$ and a $U(1)$ boson in the third side. The contribution is

$$
V_{2}^{\prime}=3 \times g^{3} \frac{2}{N}\left(f^{a x y} \cos \tilde{p}_{1} q+d^{a x y} \sin \tilde{p}_{1} q\right) \times \delta^{x b} \sin \tilde{p}_{2} q \times \delta^{y c} \sin \tilde{p}_{3}\left(q+p_{1}\right)
$$

The part that leads to divergences in (26) can be written as follows

$$
V_{2}^{\prime}=-g^{3}\left(f^{a b c} \cos \tilde{p}_{3} p_{1}+d^{a b c} \sin \tilde{p}_{3} p_{1}\right) \frac{N}{2} \frac{3}{N^{2}}
$$

Thus, collecting the two contributions $V_{2}$ and $V_{2}^{\prime}$, we find that the counter term which is needed to cancel the divergences in the 3 gluons vertex with external legs in $S U(N)$ is

$$
\delta_{1}^{(N-N-N)}=\frac{g^{2} N}{(4 \pi)^{2}} \times \frac{2}{3} \times \frac{2}{\epsilon},
$$

as in ordinary commutative Yang-Mills theory. Note that the interaction with the $U(1)^{\prime} s$ was needed to cancel the $\frac{1}{N^{2}}$ terms in (22). Another comment is that the finite contribution (21) in the $U(1)-U(1)-U(1)$ cancels in the present case.

We turn now to the renormalization of 3-gluons vertex with one external leg in $U(1)$ and two external legs in $S U(N)$ (figure 1b). The first contribution to the diagram $3 \mathrm{a}$ is when $S U(N)$ bosons circulate in the loop. We should use the Feynman rules $1 \mathrm{a}$ and $1 \mathrm{~b}$. The contribution is

$$
\begin{aligned}
& V_{3}=g^{3} \sqrt{\frac{2}{N}}\left(f^{a x y} \cos \tilde{p}_{1} q+d^{a x y} \sin \tilde{p}_{1} q\right) \times \\
& \left(f^{b y z} \cos \tilde{p}_{2} q+d^{b y z} \sin \tilde{p}_{2} q\right) \times \delta^{z x} \sin \tilde{p}_{3}\left(q+p_{1}\right)
\end{aligned}
$$


which can be simplified (by using (12), (13) and (19)) and rewritten as

$$
V_{3}=-g^{3} \sqrt{\frac{2}{N}} N\left(2-\frac{4}{N^{2}}\right) \frac{1}{4} \sin \tilde{p}_{3} p_{1} \delta^{a b}+\text { other terms }
$$

In addition there are two other diagrams which correct the $S U(N)-S U(N)-$ $U(1)$ vertex. In one of the diagrams there are two $U(1)$ bosons and one $S U(N)$ bosons which flow in the triangle (fig. 3a) and in the other there are two $S U(N)$ bosons and one $U(1)$. The two diagrams contributes the same. Their contribution is

$$
\begin{aligned}
V_{3}^{\prime} & =2 \times g^{3}\left(\frac{2}{N}\right)^{\frac{3}{2}} \sin \tilde{p}_{1} q \sin \tilde{p}_{2} q \sin \tilde{p}_{3}\left(q+p_{1}\right) \delta^{a b} \\
& =-2 \times g^{3} \sqrt{\frac{2}{N}} \frac{2}{N} \frac{1}{4} \sin \tilde{p}_{3} p_{1} \delta^{a b}+\text { other terms }
\end{aligned}
$$

The contribution $V_{3}^{\prime}$ exactly compensate the $\frac{1}{N^{2}}$ part in (30). Hence the needed counter term is

$$
\delta_{1}^{(N-N-1)}=\frac{g^{2} N}{(4 \pi)^{2}} \times \frac{2}{3} \times \frac{2}{\epsilon},
$$

exactly as (20) and (28).

The 'other terms' in eqs.(30),(31) leads to finite terms which take exactly the same form as (21).

The calculation of the 4-gluon vertices is straightforward, though tedious. Adding matter in the adjoint representation is also straightforward. The counter terms which are needed in all these cases are exactly the same as the ones which are needed in ordinary $S U(N)$ theory.

\section{Renormalizability and gauge invariance}

In the previous sections we calculated the counter terms which are needed to renormalize the theory. Since we are dealing with a gauge theory, gauge symmetry imposes some constraints on the various counter terms. In ordinary Yang-Mills theory the three gluons vertex and the four gluons vertex are multiplied by $g$ and $g^{2}$ respectively. Gauge invariance tells us that the two couplings should be the same - also at the quantum level. In the present 
case the situation is even more involved. A-priori, there are two types of propagators with different wave functions renormalization. There are also three types of vertices (even four, if we consider the $f^{a b c} \cos$ and the $d^{a b c} \sin$ parts of the $S U(N)$ vertex as two independent vertices).

Gauge invariance imposes the following relations, at one loop

$$
\delta_{1}^{(1-1-1)}-\frac{3}{2} \delta_{3}^{(1-1)}=\delta_{1}^{(N-N-N)}-\frac{3}{2} \delta_{3}^{(N-N)}=\delta_{1}^{(N-N-1)}-\delta_{3}^{(N-N)}-\frac{1}{2} \delta_{3}^{(1-1)} .
$$

We have found that in fact all $\delta_{1}^{i}$ are equal and $\delta_{3}^{i}$ are equal. Clearly, (33) is satisfied.

The calculation of the beta function is also straightforward. The $\frac{2}{\epsilon}$ in the expressions for $\delta$ should be replaced by $\log \frac{\Lambda^{2}}{\mu^{2}}$ and the beta function is computed by

$$
\beta(g)=g \mu \frac{\partial}{\partial \mu}\left(-\delta_{1}+\frac{3}{2} \delta_{3}\right)
$$

The result is

$$
\beta(g)=-\frac{g^{3}}{(4 \pi)^{2}} \frac{11}{3} N
$$

as expected [5]. Note that our result for the $U(1)$ case differs by a factor of 2 from [8] due to a different definition of the $U(1)$ coupling.

Let us comment about various limits and some special cases. In contrast to the commutative theory, where the $U(N)$ theory contains two couplings: a $U(1)$ coupling which doesn't run and an asymptotically free $S U(N)$ coupling, we showed that non-commutative theory can (and as we shall see in a moment - must) contain a single coupling. The theory is asymptotically free and the value of the beta function is independent of $\theta$. It is a bit unusual at first sight, since the commutative theory should be a limit of the non-commutative theory. However, this limit is singular. As long as $\theta$ is non-zero the $U(1)$ coupling runs, independently of the value of $\theta$ and exactly as the $S U(N)$ coupling. When $\theta$ is zero, the $U(1)$ is frozen. Thus though the Feynman rules of the non-commutative theory are smooth in $\theta$, the renormalization procedure makes the limit singular.

The planar limit is also interesting. It was suggested [2, 4] that the planar limit of the non-commutative theory is the ordinary theory. However, the planar limit of the $U(1)$ theory is not the ordinary commutative theory but rather an interacting theory and the counter term (9) is still needed. 
Similarly, the planar limit of the $U(N)$ theory is not the planar limit of the $S U(N) \times U(1)$ ordinary theory. In order to be more precise, let us give an example which clarifies the difference between the commutative and the non-commutative planar theories. Correlation functions which involve only $\operatorname{tr} F_{\mu \nu}$ would yield trivial answers in the commutative theory, since the $U(1)$ part is decoupled and free. On the other hand, such correlation functions are highly non-trivial in the planar non-commutative case.

Another remark is about the $S U(N)$ theory. It was argued in [30] (see also [31, 32 and 24 for a derivation from string theory) that the noncommutative version of this theory is not consistent, since the closure of the Moyal commutator is violated. Here we find another evidence for the inconsistency of the $S U(N)$ theory. Had we ignored the $U(1)$ part of the theory, we would have found that the value of the beta function is gauge dependent. In order to see that one should calculate the counter terms in a general gauge and to observe that a $\frac{1}{N^{2}}$ gauge dependent piece is left in the beta function of the $S U(N)$ theory.

Finally let us comment about the $\mathcal{N}=4$ theory. Since this theory is finite in the ordinary commutative case, it seems that for this specific case the limit $\theta \rightarrow 0$ is smooth. Let us focus on the planar theory first. Both the $U(1)$ and the $S U(N)$ gauge couplings take their classical value and no counter terms are needed. Therefore the $\theta \rightarrow 0$ limit is the same as the $\theta=0$ theory. The non-planar sector of the theory is more subtle. The finite UV effects are manifestly singular in $\theta$. It was suggested in [7], that in the specific case of $\mathcal{N}=4$ these contributions cancel and thus also this sector of the theory is smooth in $\theta$.

We would like to note that there is another class of theories which are UV finite [33 and maybe even smooth in $\theta$. These are the orbifold truncations of $\mathcal{N}=4$. These theories share the same planar diagrams as $\mathcal{N}=4$ [34]. Therefore, this sector of the theory is finite. The non-planar sector is anyways UV finite in non-commutative theories. Moreover, since these theories admit Bose-Fermi degeneracy, it is likely that the non-planar contributions cancel as in the $\mathcal{N}=4$ case. 


\section{ACKNOWLEDGEMENTS}

I would like to thank C. Angelantonj, I. Antoniadis, E. Gardi, F. Hassan and R. Minasian for discussions and comments. This research was supported in part by EEC under TMR contract ERBFMRX-CT96-0090. 


\section{Appendix A - Feynman rules for the non- commutative $U(N)$ Yang-Mills theory}

The non-commutative Yang-Mills action including gauge fixing and ghosts takes the following form

$$
S=\int d^{4} x \operatorname{tr}\left(-\frac{1}{2} F^{\mu \nu} \star F_{\mu \nu}+\xi\left(\partial^{\mu} A_{\mu}\right)^{2}-\bar{c} \star \partial^{\mu} D_{\mu} c+\partial^{\mu} D_{\mu} c \star \bar{c}\right)
$$

We use the Feynman-'t Hooft gauge $\xi=1$.

$$
\sim_{A, \mu}^{\mathrm{p}} \sim \mathrm{B}, v \quad-\mathrm{i} / \mathrm{p}^{2} \delta^{\mathrm{AB}}
$$

$$
\mathrm{A}, \mu \quad \cdots \cdots \cdots, \mathrm{B}, \mathrm{v} \quad \mathrm{i} / \mathrm{p}^{2} \delta^{\mathrm{AB}}
$$
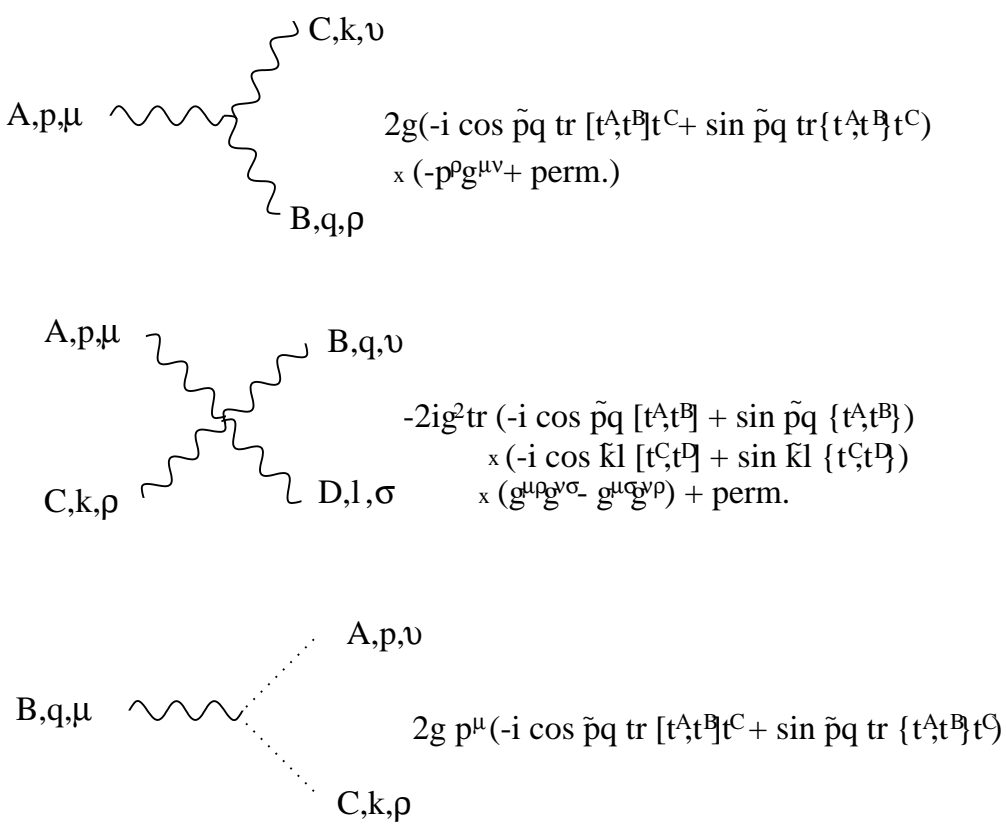

Figure 4: Feynman rules. Wavy lines and dotted lines denote gluons and ghosts, respectively. Capital letters and small letters denote $U(N)$ indices and momenta. 


\section{Appendix B - $S U(N)$ identities}

In this section we derive $S U(N)$ identities which are used in the paper.

Identity A $f^{a x y} f^{b x y}=N \delta^{a b}$.

We denote the adjoint representation by capital letters. We use $\operatorname{tr} T^{a} T^{b}=$ $N \delta^{a b}$. Also $T_{x y}^{a}=-i f^{a x y}$. Therefore $T_{x y}^{a} T_{y x}^{b}=-i f^{a x y} \times-i f^{b y x}=f^{a x y} f^{b x y}=$ $N \delta^{a b}$.

Identity $\mathbf{B} d^{a x y} d^{b x y}=\left(N-\frac{4}{N}\right) \delta^{a b}$.

$$
\operatorname{tr} t^{x} t^{x} t^{a} t^{b}=\frac{N^{2}-1}{2 N} \frac{1}{2} \delta^{a b}
$$

By using $t^{a} t^{b}=\frac{1}{2}\left(i f^{a b c} t^{c}+\frac{1}{N} \delta^{a b}+d^{a b c} t^{c}\right)$, (37) reads

$$
\begin{aligned}
& =\operatorname{tr} \frac{1}{2}\left(i f^{x a y} t^{y}+\frac{1}{N} \delta^{x a}+d^{x a y} t^{y}\right) t^{b} t^{x} \\
& =\frac{1}{2}\left(i f^{x a y}+d^{x a y}\right) \operatorname{tr} t^{y} t^{b} t^{x}+\frac{1}{4} \frac{1}{N} \delta^{x a} \delta^{b x} \\
& =\frac{1}{8}\left(f^{a x y} f^{b x y}+d^{a x y} d^{b x y}\right)+\frac{1}{4 N} \delta^{a b}
\end{aligned}
$$

Thus (37) and (38) leads to

$$
f^{a x y} f^{b x y}+d^{a x y} d^{b x y}=\left(2 N-\frac{4}{N}\right) \delta^{a b},
$$

and by using identity A, identity B is proven.

Identity $\mathrm{C}$

$$
\begin{aligned}
& f^{a x y} f^{b y z} f^{c z x}-f^{a x y} d^{b y z} d^{c z x} \\
& -d^{a x y} f^{b y z} d^{c z x}-d^{a x y} d^{b y z} f^{c z x}=2 N\left(1-\frac{3}{N^{2}}\right) f^{a b c} \\
& d^{a x y} d^{b y z} d^{c z x}-d^{a x y} f^{b y z} f^{c z x} \\
& -f^{a x y} d^{b y z} f^{c z x}-f^{a x y} f^{b y z} d^{c z x}=2 N\left(1-\frac{3}{N^{2}}\right) d^{a b c}
\end{aligned}
$$

we begin with

$$
\operatorname{tr} t^{x} t^{x} t^{a} t^{b} t^{c}=\frac{N^{2}-1}{2 N} \frac{1}{4}\left(i f^{a b c}+d^{a b c}\right)
$$


Eq. (42) can be written also as follows

$$
\begin{aligned}
& =\operatorname{tr} \frac{1}{2}\left(i f^{x a y} t^{y}+\frac{1}{N} \delta^{x a}+d^{x a y} t^{y}\right) t^{b} t^{c} t^{x} \\
& =\frac{1}{2}\left(i f^{x a y}+d^{x a y}\right) \operatorname{tr} t^{y} t^{b} t^{c} t^{x}+\frac{1}{2 N} \operatorname{tr} t^{b} t^{c} t^{a} \\
& =\frac{1}{16}\left(i f^{x a y}+d^{x a y}\right)\left(i f^{y b z}+d^{y b z}\right)\left(i f^{z c x}+d^{z c x}\right)+\frac{2}{8 N}\left(i f^{a b c}+d^{a b c}\right) .
\end{aligned}
$$

By equating (42) and (43) we arrive at

$$
\left(-i f^{a x y}+d^{a x y}\right)\left(-i f^{b y z}+d^{b y z}\right)\left(-i f^{c z x}+d^{c z x}\right)=\left(2 N-\frac{6}{N}\right)\left(i f^{a b c}+d^{a b c}\right) .
$$

The real and imaginary parts of (44) prove identity C.

\section{References}

[1] N. Seiberg and E. Witten, "String Theory and Noncommutative Geometry", JHEP 9909 (1999) 032.

[2] T. Filk, "Divergencies in a Field Theory on Quantum Space", Phys.Lett.B376 (1996) 53.

[3] A. Gonzalez-Arroyo and M. Okawa, "A Twisted Model For Large $N$ Lattice Gauge Theory", Phys. Lett. B120 (1983) 174; A. Gonzalez-Arroyo and M. Okawa, "The Twisted Eguchi-Kawai Model: A Reduced Model For Large N Lattice Gauge Thoery", Phys. Rev. D27 (1983) 2397.

[4] D. Bigatti and L. Susskind, "Magnetic fields, branes and noncommutative geometry", hep-th/9908056.

[5] S. Minwalla, M. Van Raamsdonk and N. Seiberg, "Noncommutative Perturbative Dynamics", hep-th/9912072.

[6] M. Hayakawa, "Perturbative analysis on infrared aspects of noncommutative QED on $R^{4}$ ", Phys.Lett.B478 (2000) 394; "Perturbative analysis on infrared and ultraviolet aspects of noncommutative QED on $R^{4}$ ", hepth/9912167; 
[7] A. Matusis, L. Susskind and N. Toumbas, "The IR/UV Connection in the Non-Commutative Gauge Theories", hep-th/0002075.

[8] C. P. Martin and D. Sanchez-Ruiz, "The One-loop UV Divergent Structure of U(1) Yang-Mills Theory on Noncommutative $R^{4}$, Phys.Rev.Lett. 83 (1999) 476.

[9] T. Krajewski and R. Wulkenhaar, "Perturbative quantum gauge fields on the noncommutative torus", hep-th/9903187.

[10] M. M. Sheikh-Jabbari, "One Loop Renormalizability of Supersymmetric Yang-Mills Theories on Noncommutative Two-Torus", JHEP 9906 (1999) 015.

[11] I. Chepelev and R. Roiban, "Renormalization of Quantum Field Theories on Noncommutative $R^{d}$, I. Scalars", hep-th/9911098.

[12] I. Ya. Aref'eva, D. M. Belov and A. S. Koshelev, "Two-Loop Diagrams in Noncommutative $\phi_{4}^{4}$ theory", Phys.Lett.B476 (2000) 431.

[13] H. Grosse, T. Krajewski and R. Wulkenhaar, "Renormalization of noncommutative Yang-Mills theories: A simple example", hep-th/0001182.

[14] I. Ya. Aref'eva, D. M. Belov and A. S. Koshelev, "A Note on UV/IR for Noncommutative Complex Scalar Field", hep-th/0001215.

[15] M. V. Raamsdonk and N. Seiberg, "Comments on Noncommutative Perturbative Dynamics", JHEP 0003 (2000) 035.

[16] F. Ardalan and N. Sadooghi, "Axial Anomaly in Non-Commutative QED on $R^{4}$, hep-th/0002143.

[17] J. M. Gracia-Bondia and C. P. Martin, "Chiral Gauge Anomalies on Noncommutative $R^{4}$ ", Phys.Lett.B479 (2000) 321.

[18] C.-S. Chu, "Induced Chern-Simons and WZW action in Noncommutative Spacetime", hep-th/0003007.

[19] I. Ya. Aref'eva, D. M. Belov, A. S. Koshelev and O. A. Rytchkov, "UV/IR Mixing for Noncommutative Complex Scalar Field Theory, II (Interaction with Gauge Fields)", hep-th/0003176. 
[20] A. Rajaraman and M. Rozali, "Noncommutative Gauge Theory, Divergences and Closed Strings", hep-th/0003227.

[21] K. Furuta and T. Inami, "Ultraviolet Property of Noncommutative WessZumino-Witten Model", hep-th/0004024.

[22] A. A. Bichl, J. M. Grimstrup, V. Putz and M. Schweda, "Perturbative Chern-Simons Theory on Noncommutative $R^{3}$ ", hep-th/0004071.

[23] J. Gomis and T. Mehen, "Space-Time Noncommutative Field Theories and Unitarity", hep-th/0005129.

[24] O. Andreev and H. Dorn, "Diagrams of Noncommutative $\phi^{3}$ Theory from String Theory", hep-th/0003113.

[25] Y. Kiem and S. Lee, "UV/IR Mixing in Noncommutative Field Theory via Open String Loops", hep-th/0003145.

[26] A. Bilal, C.-S. Chu and R. Russo, "String Theory and Noncommutative Field Theories at One Loop", hep-th/0003180.

[27] K. Gomis, K. Kleban, T. Mehen, M. Rangamani and S. Shenker, "Noncommutative Gauge Dynamics From The String Worldsheet", hepth/0003215.

[28] H. Liu and J. Michelson, "Stretched Strings in Noncommutative Field Theory", hep-th/0004013.

[29] C.-S. Chu, R. Russo and S. Sciuto, "Multiloop String Amplitudes with $B$-Field and Noncommutative QFT", hep-th/0004183.

[30] K. Matsubara, "Restrictions on Gauge Groups in Noncommutative Gauge Theory", hep-th/0003294.

[31] J. Madore, S. Schraml, P. Schupp and J. Wess, "Gauge Theory on Noncommutative Spaces", hep-th/0001203.

[32] S. Terashima, "A Note on Superfields and Noncommutative Geometry", hep-th/0002119. 
[33] A. Armoni, "A Note on Non-Commutative Orbifold Field Theories", JHEP 0003 (2000) 033.

[34] M. Bershadsky and A. Johansen, "Large $N$ limit of orbifold field theories", Nucl.Phys. B536 (1998) 141. 